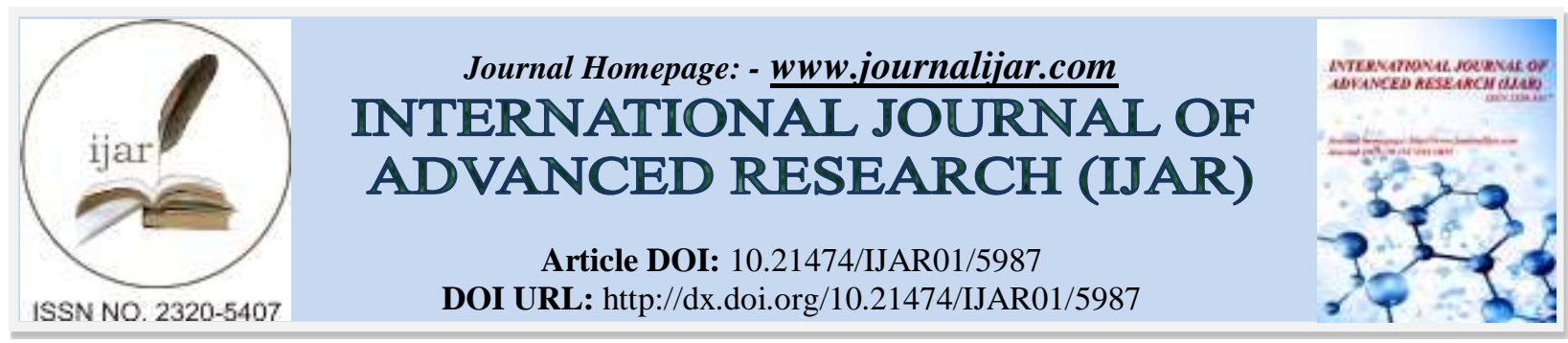

RESEARCH ARTICLE

\title{
EFFECT OF POST HARVEST TREATMENTS ON QUALITY AND SHELF LIFE OF BANANA CV. GRAND NAINE.
}

Manroop Kaur and Amarjeet Kaur.

Department of Agriculture Khalsa College Amritsar-143001.

\section{Manuscript Info}

Manuscript History

Received: 06 October 2017

Final Accepted: 08 November 2017

Published: December 2017

Key words:-

Banana, Grand Naine, Ripening, Ethrel,

Firmness, Peel ratio, Pulp ratio,Sugars,Acidity.

\section{Abstract}

The present investigation entitled Effect of various ripening agents on physical characteristics of banana cv. Grand Naine was conducted in the laboratory of the Department of Horticulture, Khalsa College, Amritsar during 2016-2017. The experiment comprised of six postharvest treatments viz., ethrel 300 ppm, ethrel 400 ppm, ethrel 600 ppm, cold water + ethrel 200 ppm, hot water + ethrel 200 ppm and control. Changes in different physical characteristics of banana were studied at 2 days interval during storage for 8 days. The two-factor experiment was laid out in Completely Randomized Design (CRD) with three replications. Results of the study revealed that marked variations were recorded among the post harvest treatments on ripening of banana. The highest fruit color (7), pulp to peel ratio $(2.41 \%)$, fruit taste (9.10), physiological loss in weight(5.9\%) and minimum fruit firmness $\left(6.00 \mathrm{~kg} / \mathrm{cm}^{2}\right)$ was observed in the treatment of ethrel 600 ppm. Among bio-chemical characters the highest moisture content in pulp $(76.60 \%)$, total soluble solids $(21.97 \%)$, total sugars $(18.09 \%)$, reducing sugars $(12.10 \%)$ and minimum titratable acidity $(0.18 \%)$ and starch content $(3.01 \%)$ were also observed in the treatment of ethrel $600 \mathrm{ppm}$

Copy Right, IJAR, 2017,. All rights reserved.

\section{Introduction:-}

Banana(Musa sp.) is one of the ancient fruit of the world belonging to the family Musaceae. It is also known as apple of the paradise and is the tallest of the herbaceous plants with pseudostem (Rahman and Kabir 2006). It is also cultivated on a commercial scale in India, China, Phillipines, Mexico and Columbia. It is equally liked by all age group of people because it is easily digestible and palatable. From nutritional point of view, it has high calorific and nutritional values. It contains carbohydrates, crude fiber, proteins, fat, ash, phosphorous, iron, $\beta$ - carotene, riboflavin, niacin and ascorbic acid (Akter et al 2013). The banana fruit can be eaten raw or cooked. It can be processed into flour and can be fermented for the production of beverages such as banana juice, vinegar and wine (Pillay and Tripathi 2007).Grand Naine is a popular variety grown in all the export oriented countries of Asia, South America and Africa.It is a superior selection of Giant Cavendish variety which was introduced in India in 1990.It has high productivity and is more vigorous and robust with well spaced hands, bigger sized fingers and heavy bunches (Singh and Chundawat 2002). Banana is a climacteric fruit showing an increase in respiration rate resulting in development of color, flavor and aroma. Banana is harvested mature but in unripe condition and is subsequently allowed to ripen further. In natural conditions, they ripen slowly, leading to high weight loss, desiccation, uneven ripening and failure to develop good color and aroma. Hence marketable quality gets deteriorated. Therefore to 
reduce the post harvest losses bananas are harvested green and are normally artificially ripened with the use of ripening agents( Temi et al 2014). These include ethylene gas, ethephon, ethrel which helps in early and uniform ripening of banana fruits with good color development and taste. Ethrel treatment of fruits results in high marketable fruit, minimum spoilage percentage and higher peel : pulp ratio (Pendharkar et al 2011 ).

\section{Materials and methods:-}

The present investigation entitled effect of various ripening agents on the physical characters banana cv. Grand Naine was conducted at the laboratory of department of horticulture, Khalsa College, Amritsar during the year 201617. The material used for the present experiment were freshly harvested mature banana bunches of cv. Grand Naine. The bananas used in the experiment were collected from the private orchard of Mewa Singh of Village Kulaar, Ludhiana. The experiment was conducted in Completely Randomized Design with three replications and six treatments viz., $\mathrm{T}_{1}$ : Control, $\mathrm{T}_{2}$ : Ethrel $300 \mathrm{ppm}$ (3 minutes dip) $\mathrm{T}_{3}$ : Ethrel $400 \mathrm{ppm}$ (3 minutes dip), $\mathrm{T}_{4}$ : Ethrel 600 ppm (3 minutes dip), $\mathrm{T}_{5}$ : Cold water +ethrel $200 \mathrm{ppm}\left(3\right.$ minutes dip), $\mathrm{T}_{6}:$ Hot water $\left(50 \pm 2{ }^{\circ} \mathrm{C}\right)+\mathrm{ethrel} 200 \mathrm{ppm}$ (3 minutes dip). After the application of treatments, the fruits were kept on newspapers on the laboratory table at room temperature. Each treatment comprised of 20 fingers. Detailed observations were recorded at $2^{\text {nd }}, 4^{\text {th }}, 6^{\text {th }}$ and $8^{\text {th }}$ days for all physical and bio-chemical parameters like fruit firmness, physiological loss in weight, fruit taste, pulp to peel ratio, fruit color, TSS, sugars and starch respectively.

\section{Results and discussion:-}

The results showed reduction in fruit firmness steadily over a period of 8 days. Untreated control fruits were hard with firmness $\left(7.10 \mathrm{~kg} / \mathrm{cm}^{2}\right)$ even after 8 days and remained partially ripened and showed maximum firmness during the whole storage period. After 6 days of storage fruits treated with ethrel $600 \mathrm{ppm}$ were the least firm $\left(6.53 \mathrm{~kg} / \mathrm{cm}^{2}\right.$ ).The data showed descending trend in fruit firmness with an increase in the concentration of ethrel. Softening of fruits is related to a change in cell wall components and starch degradation. With the advance in ripening there may be breakdown of the cell walls, reduction in the cohesion of middle lamella due to solubilization of the pectic substances and movement of water from the skin to the flesh as a result of osmosis, all of which result in softening of the fruits (Zewter et al 2012). The research findings of Kulkarni et al (2012) in Robusta and Mahajan et al (2010) in Grand Naine banana cultivars are in agreement with the present studies.

The highest physiological loss in weight was observed with ethrel $600 \mathrm{ppm}(5.9 \%)$ on the $8^{\text {th }}$ day of storage which resulted in shrivelling, softening and over-ripening of fruits. Ethrel $400 \mathrm{ppm}$ and $300 \mathrm{ppm}$ recorded (3.5\%) and (3.9 $\%$ ) weight loss respectively during the ripening period of $8^{\text {th }}$ day leading to adequate ripening and softening of fruits followed by cold water treatment + ethrel $200 \mathrm{ppm}$ and hot water treatment $\left(50^{\circ} \pm 2^{\circ} \mathrm{C}\right)+\mathrm{ethrel} 200 \mathrm{ppm}$ with loss in weight of $(3.3 \%)$ and $(3.0 \%)$. The treatments of control observed the physiological loss in weight $(2.9 \%)$. In all treatments the percentage weight loss increased with the storage time and ripening progressed, which possibly resulted from transpiration and respiration of the fruits. Energy produced from the respiration process in the form of heat is released from the fruit by evaporation of water causing a weight loss (Dharmasena and Kumari 2005). Loss in weight by various treatments also has also been reported by Mahajan et al (2010) in banana cv. Grand Naine and Venktasubbaiah et al (2013) and Kumar (2006) also reported the same in banana fruits.

The fruit taste was affected by the post harvest treatments of ethrel. Maximum score of fruit taste was observed in the treatment of ethrel $600 \mathrm{ppm}$ on $6^{\text {th }}$ day of storage which was at par with ethrel 400 ppm (9.10) after 8 days of storage. The treatment of ethrel $300 \mathrm{ppm}$ and cold water + ethrel $300 \mathrm{ppm}$ recorded the fruit taste (8.99) which was followed by hot water + ethrel $200 \mathrm{ppm}$ treatment(8.95). The untreated fruits were not in edible condition until after 6 days of storage indicating long period required for ripening. The increase in taste during ripening was due to the increase in certain volatile compounds which acted as a precursor for taste in the fruit cells. The findings of Kulkarni et al (2011) in banana cv. Robusta, Mahajan et al (2010) in cv. Grand Naine are in support with the present results. Highest pulp to peel ratio (2.41) was recorded in the treatment of ethrel 600 ppm followed by ethrel 400 ppm (2.23 ). Minimum value of pulp to peel ratio was noticed under control (1.64). The increase in the pulp to peel ratio might be due to the change in sugar concentration in the pulp compared to the peel thus contributing to different change in osmotic pressure. Water might be lost from the peel of banana both by transpiration and osmosis due to which the peel weight was reduced and pulp to peel ratio increased. The increase in pulp to peel ratio during ripening was observed by Simmonds (1996) and Krishnamurthy (1993). 
It evident from the Table1 that ethrel had considerable effect on peel color of banana fruits. The fastest color change was observed in ethrel $600 \mathrm{ppm}$ treatment and reached at full yellow color with brown spots over it on $8^{\text {th }}$ day with scoring of (7.00). On the same day, fruits treated with ethrel $400 \mathrm{ppm}$ scored (6.33). Ethrel $300 \mathrm{ppm}$ scored (6.00), cold water + ethrel $200 \mathrm{ppm}$ (5.66) and hot water treatment + ethrel $200 \mathrm{ppm}$ (5.33) on $8^{\text {th }}$ day of storage. The control fruits showed the scores (4.33). The conversion of green color of the peel into yellow might be as a result of chlorophyll degradation which acted as an indicator of senescence that was enhanced by high rate of respiration which in turn was regulated by temperature, ethylene, $\mathrm{O}_{2}, \mathrm{CO}_{2}$ gasses (Pinto et al.2004).

Post harvest treatments with different chemicals significantly affected the TSS content of banana fruits. Maximum TSS $\left(22.80^{\circ}\right.$ Brix $)$ was recorded in the treatment of ethrel $600 \mathrm{ppm}$ followed by ethrel $400 \mathrm{ppm}\left(21.97^{\circ} \mathrm{Brix}\right) \mathrm{TSS}$ after 6 days of storage while ethrel $300 \mathrm{ppm}$ and cold water + ethrel $200 \mathrm{ppm}$ showed TSS of $\left(21.65{ }^{\circ} \mathrm{Brix}\right)$ and $\left(21.19{ }^{\circ}\right.$ Brix) respectively after 6 of storage. The treatment of hot water +ethrel $200 \mathrm{ppm}$ showed the TSS of (21.00 $\left.{ }^{\circ} \mathrm{Brix}\right)$ which was followed by control $\left(20.61{ }^{\circ} \mathrm{Brix}\right)$ treatment. The present results are in agreement with the report of Dharmasena and Kumari (2005) and Salvador et al (2006) in banana. The increase in TSS content of fruits might be an indication of high respiration rate and ripening stages, thereby resulting in quality deterioration with the onset of senescence. The results are in agreement with Kulkarni et al (2011). The findings of pendharkar et al (2011) in Grand Naine are also in support with the present studies.

It is demonstrated from the present studies that during the whole storage period moisture content in pulp of banana increased. Ethrel treatments had marginal effect on moisture content. The highest moisture content (76.60 \%) was recorded in ethrel $600 \mathrm{ppm}$. Ethrel treatment $400 \mathrm{ppm}$ showed $(74.30 \%)$ which was followed by ethrel $300 \mathrm{ppm}$ (74.50\%), cold water + ethrel 200ppm (73.80) after 8 days of storage. The treatment of hot water + ethrel $200 \mathrm{ppm}$ recorded moisture content (73.30). The treatments were further followed by control treatment $(72.90 \%)$. The increase in pulp moisture content during ripening might be due to the carbohydrate breakdown and osmotic transfer from peel to pulp. The findings of Kulkarni et al (2011) in banana cv. Robusta are in accordance with the present results.

Minimum acidity $(0.36 \%, 0.29 \%, 0.23 \%$ and $0.18 \%)$ was observed in ethrel 600 ppm during 8 days of storage period. Optimum range of acidity $(0.26 \%)$ was observed in the ethrel treatment $400 \mathrm{ppm}$ after 6 days of storage. The decrease in titratable acidity during storage might be due to the utilization of organic acids in respiration process and other bio-degradable reactions, glycolytic pathways or might have been used in respiration or both. Earlier findings of Mahajan et al (2010) in banana cv. Grand Naine, Kulkarni et al (2011) in Robusta banana and Venkatasubbaiah et al (2013) in Grand Naine banana also obtained the similar results. The present studies are also in line with the findings of Pendharkar et al (2011) in banana cv. Grand Naine.

The data on the effect of post harvest application of ethrel treatments on total sugars of banana cv. Grand Naine depicted that significantly higher total sugars $(18.09 \%)$ were analysed from the fruits treated with ethrel $600 \mathrm{ppm}$ followed by ethrel $400 \mathrm{ppm}$ with $(17.82 \%)$ and ethrel $300 \mathrm{ppm}$ with $(17.79 \%)$ total sugars. Treatment of cold water treatment +ethrel $200 \mathrm{ppm}$ registered (17.59\%) total sugars followed by hot water +ethrel $200 \mathrm{ppm}(17.10 \%)$, and control (16.98\%). The observed increment in the amount of total sugars might be due to the conversion of starch into sugars due to respiratory metabolic pathways in starch hydrolysis. The increase in sugars in ethrel $600 \mathrm{ppm}$ might be due to the faster ripening process which converted starch into sugar. The research findings of Mahajan $e t$ al (2010) and Pendharkar et al (2011) in banana cv. Grand Naine are in line with the present findings.

Data regarding effect of post harvest treatments of ethrel on reducing sugars of banana cv. Grand Naine are presented in Table 1. From the perusal of the data it is clear that ethrel exerted a significant effect on the reducing sugars depicting the highest percentage of reducing sugars recorded in the fruits treated with ethrel $600 \mathrm{ppm}(12.10$ $\%)$ followed by ethrel $400 \mathrm{ppm}(11.45 \%)$ and ethrel 300ppm $(11.35 \%)$. Cold water + ethrel $200 \mathrm{ppm}$ recorded the reducing sugars of $(11.20 \%)$ which was followed by hot water + ethrel $200 \mathrm{ppm}(11.05 \%)$, and control treatment (10.30 \%). The findings of Morrelli and Kader (2002), Pendharkar et al (2011) and Mahajan et al (2010) in banana cv. Grand Naine are in confirmation with the present studies.

Starch level decreased in all the treatments as ripening advanced. However decrease was more rapid with ethrel 600 ppm $(3.01 \%)$ followed by ethrel $400 \mathrm{ppm}(5.07 \%)$ and ethrel $300 \mathrm{ppm}(6.01 \%)$ after 8 days of storage. Cold water + ethrel $200 \mathrm{ppm}$ recorded the starch content of (6.44\%) which was closely followed by hot water treatment + ethrel $200 \mathrm{ppm}(6.58 \%)$. Maximum starch content was found under control treatment (6.95\%). Ripening resulted 
in decline of starch content which could be attributed to the increased activity of amylase and other enzymes resulting in gluconeogenesis. Deepak et al (2008), (Gowda and Huddar 2001). The findings of Venktasubbaiah et al (2013) in banana cv. Grand Naine are in agreement with the present results.

Table 1:- Effect of post harvest treatments on physical characteristics of banana cv. Grand Naine

\begin{tabular}{|c|c|c|c|c|c|c|c|c|c|c|c|c|c|c|c|c|c|c|c|c|}
\hline \multirow[t]{2}{*}{$\begin{array}{l}\text { Treatm } \\
\text { ents }\end{array}$} & \multicolumn{4}{|c|}{ Fruit firmness } & \multicolumn{4}{|c|}{$\begin{array}{c}\text { Physiological loss } \\
\text { in weight }\end{array}$} & \multicolumn{4}{|c|}{ Pulp to peel ratio } & \multicolumn{4}{|c|}{ Fruit taste } & \multicolumn{4}{|c|}{ Fruit colour } \\
\hline & $\begin{array}{c}2^{\text {n }} \\
\text { d } \\
\text { da } \\
y\end{array}$ & $\begin{array}{c}4^{\text {th }} \\
\text { da } \\
y\end{array}$ & $\begin{array}{c}6^{\text {th }} \\
\text { da } \\
y\end{array}$ & $\begin{array}{c}8^{\text {th }} \\
\text { da } \\
y\end{array}$ & $\begin{array}{c}2^{\text {n }} \\
\text { d } \\
\text { da } \\
y\end{array}$ & $\begin{array}{l}4^{\text {th }} \\
\text { da } \\
y\end{array}$ & $\begin{array}{c}6^{\text {th }} \\
\text { da } \\
y\end{array}$ & $\begin{array}{c}8^{\text {th }} \\
\text { da } \\
y\end{array}$ & $\begin{array}{c}2^{n} \\
d \\
d a \\
y\end{array}$ & $\begin{array}{c}4^{\text {th }} \\
\text { da } \\
y\end{array}$ & $\begin{array}{c}6^{\text {th }} \\
\text { da } \\
y\end{array}$ & $\begin{array}{c}8^{\text {th }} \\
\text { da } \\
y\end{array}$ & $\begin{array}{c}2^{n} \\
d \\
d a \\
y\end{array}$ & $\begin{array}{c}4^{\text {th }} \\
\text { da } \\
y\end{array}$ & $\begin{array}{c}6^{\text {th }} \\
\text { da } \\
y\end{array}$ & $\begin{array}{c}8^{\text {th }} \\
\text { da } \\
y\end{array}$ & $\begin{array}{c}2^{\mathrm{n}} \\
\mathrm{d} \\
\mathrm{da} \\
\mathrm{y}\end{array}$ & $\begin{array}{c}4^{\text {th }} \\
\text { da } \\
y\end{array}$ & $\begin{array}{l}6^{\text {th }} \\
\text { da } \\
y\end{array}$ & $\begin{array}{c}8^{\text {th }} \\
\text { da } \\
y\end{array}$ \\
\hline $\begin{array}{l}\mathrm{T}_{1}: \\
\text { Control }\end{array}$ & $\begin{array}{l}8 . \\
49\end{array}$ & $\begin{array}{l}8 . \\
21\end{array}$ & $\begin{array}{l}7 . \\
73\end{array}$ & $\begin{array}{l}7 . \\
10\end{array}$ & $\begin{array}{l}1 . \\
21\end{array}$ & $\begin{array}{l}1 . \\
96\end{array}$ & $\begin{array}{l}2 . \\
40\end{array}$ & $\begin{array}{l}2 . \\
90\end{array}$ & $\begin{array}{l}1 . \\
24\end{array}$ & $\begin{array}{l}1 . \\
38\end{array}$ & $\begin{array}{l}1 . \\
52\end{array}$ & $\begin{array}{l}1 . \\
64\end{array}$ & $\begin{array}{l}0 . \\
00\end{array}$ & $\begin{array}{l}6 . \\
72\end{array}$ & $\begin{array}{l}8 . \\
75\end{array}$ & $\begin{array}{l}8 . \\
83\end{array}$ & $\begin{array}{l}1 . \\
00\end{array}$ & $\begin{array}{l}2 . \\
00\end{array}$ & $\begin{array}{l}2 . \\
67\end{array}$ & $\begin{array}{l}4 . \\
33\end{array}$ \\
\hline $\begin{array}{l}\mathrm{T}_{2}: \\
\text { Ethrel } \\
300 \\
\mathrm{ppm}\end{array}$ & $\begin{array}{l}8 . \\
38\end{array}$ & $\begin{array}{l}8 . \\
04\end{array}$ & $\begin{array}{l}7 . \\
53\end{array}$ & $\begin{array}{l}6 . \\
84\end{array}$ & $\begin{array}{l}1 . \\
32\end{array}$ & $\begin{array}{l}2 . \\
40\end{array}$ & $\begin{array}{c}3 . \\
00\end{array}$ & $\begin{array}{l}3 . \\
50\end{array}$ & $\begin{array}{l}1 . \\
33\end{array}$ & $\begin{array}{l}1 . \\
62\end{array}$ & $\begin{array}{l}1 . \\
83\end{array}$ & $\begin{array}{l}2 . \\
17\end{array}$ & $\begin{array}{c}0 . \\
00\end{array}$ & $\begin{array}{l}6 . \\
90\end{array}$ & $\begin{array}{l}8 . \\
83\end{array}$ & $\begin{array}{l}8 . \\
99\end{array}$ & $\begin{array}{l}1 . \\
33\end{array}$ & $\begin{array}{l}3 . \\
66\end{array}$ & $\begin{array}{l}4 . \\
00\end{array}$ & $\begin{array}{c}6 . \\
00\end{array}$ \\
\hline $\begin{array}{l}\mathrm{T}_{3}: \\
\text { Ethrel } \\
400 \\
\mathrm{ppm}\end{array}$ & $\begin{array}{l}8 . \\
24\end{array}$ & $\begin{array}{l}7 . \\
82\end{array}$ & $\begin{array}{l}7 . \\
20\end{array}$ & $\begin{array}{l}6 . \\
21\end{array}$ & $\begin{array}{l}1 . \\
60\end{array}$ & $\begin{array}{l}2 . \\
80\end{array}$ & $\begin{array}{l}3 . \\
50\end{array}$ & $\begin{array}{l}3 . \\
90\end{array}$ & $\begin{array}{l}1 . \\
38\end{array}$ & $\begin{array}{l}1 . \\
63\end{array}$ & $\begin{array}{l}1 . \\
86\end{array}$ & $\begin{array}{l}2 . \\
23\end{array}$ & $\begin{array}{l}0 . \\
00\end{array}$ & $\begin{array}{l}7 . \\
32\end{array}$ & $\begin{array}{l}9 . \\
00\end{array}$ & $\begin{array}{l}9 . \\
10\end{array}$ & $\begin{array}{l}1 . \\
66\end{array}$ & $\begin{array}{l}3 . \\
33\end{array}$ & $\begin{array}{l}4 . \\
33\end{array}$ & $\begin{array}{l}6 . \\
33\end{array}$ \\
\hline $\begin{array}{l}\text { T4: } \\
\text { Ethrel } \\
600 \\
\text { ppm }\end{array}$ & $\begin{array}{l}7 . \\
79\end{array}$ & $\begin{array}{l}7 . \\
25\end{array}$ & $\begin{array}{c}6 . \\
53\end{array}$ & $\begin{array}{c}6 . \\
00\end{array}$ & $\begin{array}{c}1 . \\
80\end{array}$ & $\begin{array}{c}3 . \\
10\end{array}$ & $\begin{array}{l}4 . \\
30\end{array}$ & $\begin{array}{c}5 . \\
90\end{array}$ & $\begin{array}{l}1 . \\
43\end{array}$ & $\begin{array}{l}1 . \\
84\end{array}$ & $\begin{array}{c}1 . \\
98\end{array}$ & $\begin{array}{l}2 . \\
41\end{array}$ & $\begin{array}{c}0 . \\
00\end{array}$ & $\begin{array}{l}7 . \\
82\end{array}$ & $\begin{array}{l}9 . \\
36\end{array}$ & $\begin{array}{l}9 . \\
20\end{array}$ & $\begin{array}{c}. \\
00\end{array}$ & $\begin{array}{l}4 . \\
00\end{array}$ & $\begin{array}{c}5 . \\
00\end{array}$ & $\begin{array}{c}7 . \\
00\end{array}$ \\
\hline $\begin{array}{l}\mathrm{T}_{5}: \text { Cold } \\
\text { water+e } \\
\text { theral } \\
200 \\
\text { ppm }\end{array}$ & $\begin{array}{l}8 . \\
41\end{array}$ & $\begin{array}{c}8 . \\
11\end{array}$ & $\begin{array}{l}7 . \\
62\end{array}$ & $\begin{array}{c}6 . \\
99\end{array}$ & $\begin{array}{c}1 . \\
30\end{array}$ & $\begin{array}{c}2 . \\
30\end{array}$ & $\begin{array}{c}3 . \\
00\end{array}$ & $\begin{array}{c}3 . \\
30\end{array}$ & $\begin{array}{c}1 . \\
30\end{array}$ & $\begin{array}{c}1 . \\
50\end{array}$ & $\begin{array}{l}1 . \\
67\end{array}$ & $\begin{array}{c}1 . \\
71\end{array}$ & $\begin{array}{c}0 . \\
00\end{array}$ & $\begin{array}{c}6 . \\
86\end{array}$ & $\begin{array}{l}8 . \\
81\end{array}$ & $\begin{array}{c}8 . \\
99\end{array}$ & $\begin{array}{c}1 . \\
00\end{array}$ & $\begin{array}{l}2 . \\
66\end{array}$ & $\begin{array}{l}3 . \\
67\end{array}$ & $\begin{array}{l}5 . \\
66\end{array}$ \\
\hline $\begin{array}{l}\mathrm{T}_{6}: \text { Hot } \\
\text { water+ } \\
\text { etheral } \\
200 \\
\text { ppm }\end{array}$ & $\begin{array}{l}8 . \\
42\end{array}$ & $\begin{array}{c}8 . \\
12\end{array}$ & $\begin{array}{l}7 . \\
64\end{array}$ & $\begin{array}{c}7 . \\
05\end{array}$ & $\begin{array}{l}1 . \\
28\end{array}$ & $\begin{array}{c}2 . \\
20\end{array}$ & $\begin{array}{c}2 . \\
90\end{array}$ & $\begin{array}{c}3 . \\
00\end{array}$ & $\begin{array}{c}1 . \\
30\end{array}$ & $\begin{array}{l}1 . \\
48\end{array}$ & $\begin{array}{l}1 . \\
62\end{array}$ & $\begin{array}{c}1 . \\
70\end{array}$ & $\begin{array}{c}0 . \\
00\end{array}$ & $\begin{array}{l}6 . \\
84\end{array}$ & $\begin{array}{l}8 . \\
80\end{array}$ & $\begin{array}{l}8 . \\
95\end{array}$ & $\begin{array}{c}1 . \\
00\end{array}$ & $\begin{array}{l}2 . \\
33\end{array}$ & $\begin{array}{l}3 . \\
33\end{array}$ & $\begin{array}{l}5 . \\
33\end{array}$ \\
\hline $\begin{array}{l}\text { CD @ } \\
5 \% \\
\text { level }\end{array}$ & $\begin{array}{c}0 . \\
06\end{array}$ & $\begin{array}{c}0 . \\
05\end{array}$ & $\begin{array}{c}0 . \\
09\end{array}$ & $\begin{array}{c}0 . \\
07\end{array}$ & $\begin{array}{c}0 . \\
05\end{array}$ & $\begin{array}{c}0 . \\
15\end{array}$ & $\begin{array}{c}0 . \\
50\end{array}$ & $\begin{array}{c}0 . \\
30\end{array}$ & $\begin{array}{c}0 . \\
05\end{array}$ & $\begin{array}{c}0 . \\
08\end{array}$ & $\begin{array}{c}0 . \\
07\end{array}$ & $\begin{array}{c}0 . \\
06\end{array}$ & $\begin{array}{c}0 . \\
00\end{array}$ & $\begin{array}{c}0 . \\
80\end{array}$ & $\begin{array}{c}0 . \\
14\end{array}$ & $\begin{array}{l}\mathrm{N} \\
\mathrm{S}\end{array}$ & $\begin{array}{c}0 . \\
60\end{array}$ & $\begin{array}{c}0 . \\
80\end{array}$ & $\begin{array}{c}0 . \\
80\end{array}$ & $\begin{array}{c}0 . \\
80\end{array}$ \\
\hline
\end{tabular}

Table 2:- Effect of post harvest treatments on bio-chemical characteristics of banana cv. Grand Naine.

\begin{tabular}{|c|c|c|c|c|c|c|c|c|c|c|c|c|c|c|c|c|c|c|c|c|c|}
\hline \multirow[t]{2}{*}{$\begin{array}{l}\text { Treat } \\
\text { ment } \\
\text { s }\end{array}$} & \multicolumn{4}{|c|}{$\begin{array}{l}\text { Total soluble } \\
\text { solids (\%) }\end{array}$} & \multicolumn{4}{|c|}{$\begin{array}{c}\text { Moisture } \\
\text { content of pulp } \\
(\%)\end{array}$} & \multicolumn{4}{|c|}{$\begin{array}{l}\text { Titratable } \\
\text { acidity }(\%)\end{array}$} & \multicolumn{4}{|c|}{ Total sugars (\%) } & \multicolumn{4}{|c|}{$\begin{array}{l}\text { Reducing } \\
\text { sugars }(\%)\end{array}$} & $\begin{array}{l}\text { Sta } \\
\text { rch } \\
\text { con }\end{array}$ \\
\hline & $\begin{array}{c}\mathbf{2}^{\mathbf{n}} \\
\mathbf{d} \\
\mathbf{d a} \\
\mathbf{y}\end{array}$ & $\begin{array}{c}4^{\text {th }} \\
\text { da } \\
y\end{array}$ & $\begin{array}{c}6^{\text {th }} \\
\text { da } \\
y\end{array}$ & $\begin{array}{c}8^{\text {th }} \\
\text { da } \\
y\end{array}$ & $\begin{array}{c}2^{\mathbf{n}} \\
\mathrm{d} \\
\mathrm{da} \\
\mathbf{y}\end{array}$ & $\begin{array}{l}4^{t} \\
h \\
d \\
a \\
y\end{array}$ & $\begin{array}{c}6^{\text {th }} \\
\text { da } \\
y\end{array}$ & $\begin{array}{l}\text { d } \\
\mathbf{a} \\
\mathbf{y}\end{array}$ & $\begin{array}{l}2^{\mathbf{n}} \\
\mathbf{d} \\
\mathbf{d} \\
\mathbf{a} \\
\mathbf{y}\end{array}$ & $\begin{array}{l}4^{t} \\
\mathrm{~d} \\
\mathbf{a} \\
\mathbf{y}\end{array}$ & $\begin{array}{l}\mathbf{6}^{\mathrm{t}} \\
\mathrm{h} \\
\mathrm{d} \\
\mathrm{a} \\
\mathrm{y}\end{array}$ & $\begin{array}{l}8^{t} \\
h \\
d \\
a \\
y\end{array}$ & $\begin{array}{l}2^{\mathbf{n}} \\
\mathbf{d} \\
\mathbf{d} \\
\mathbf{a} \\
\mathbf{y}\end{array}$ & $\begin{array}{c}4^{\text {th }} \\
\text { da } \\
y\end{array}$ & $\begin{array}{c}6^{\text {th }} \\
\text { da } \\
y\end{array}$ & $\begin{array}{c}8^{\text {th }} \\
\text { da } \\
y\end{array}$ & $\begin{array}{l}2^{\mathbf{n}} \\
\mathbf{d} \\
\mathbf{d} \\
\mathbf{a} \\
\mathbf{y}\end{array}$ & $\begin{array}{l}\mathbf{4}^{\mathrm{t}} \\
\mathrm{h} \\
\mathrm{d} \\
\mathbf{a} \\
\mathbf{y}\end{array}$ & $\begin{array}{l}\mathbf{6}^{\mathbf{t}} \\
\mathbf{h} \\
\mathrm{d} \\
\mathbf{a} \\
\mathbf{y}\end{array}$ & $\begin{array}{c}8^{\text {th }} \\
\text { da } \\
y\end{array}$ & $\begin{array}{l}2^{\text {nd }} \\
\text { day } \\
4^{\text {th }} \\
\text { day } \\
6^{\text {th }} \\
\text { day }\end{array}$ \\
\hline
\end{tabular}




\begin{tabular}{|c|c|c|c|c|c|c|c|c|c|c|c|c|c|c|c|c|c|c|c|c|c|}
\hline & & & & & & & & & & & & & & & & & & & & & $\begin{array}{l}8^{\text {th }} \\
\text { day }\end{array}$ \\
\hline $\begin{array}{l}\mathrm{T}_{1}: \\
\text { Contr } \\
\text { ol }\end{array}$ & $\begin{array}{l}11 \\
.9 \\
8\end{array}$ & $\begin{array}{l}18 \\
.6 \\
4\end{array}$ & $\begin{array}{l}20 \\
.5 \\
5\end{array}$ & $\begin{array}{l}20 \\
.6 \\
1\end{array}$ & $\begin{array}{l}70 \\
.0 \\
6\end{array}$ & $\begin{array}{l}7 \\
2 . \\
3\end{array}$ & $\begin{array}{l}72 \\
.6\end{array}$ & $\begin{array}{l}7 \\
2 . \\
9\end{array}$ & $\begin{array}{l}0 . \\
5\end{array}$ & $\begin{array}{l}0 . \\
4 \\
2\end{array}$ & $\begin{array}{l}0 . \\
3 \\
9\end{array}$ & $\begin{array}{l}0 . \\
3 \\
0\end{array}$ & $\begin{array}{l}4 . \\
5 \\
2\end{array}$ & $\begin{array}{l}7 . \\
99\end{array}$ & $\begin{array}{l}10 \\
.2 \\
9\end{array}$ & $\begin{array}{l}16 \\
.7 \\
8\end{array}$ & $\begin{array}{l}4 . \\
3 \\
3\end{array}$ & $\begin{array}{l}6 . \\
3 \\
8\end{array}$ & $\begin{array}{l}8 . \\
1 \\
7\end{array}$ & $\begin{array}{l}10 \\
.3\end{array}$ & $\begin{array}{l}17 . \\
42 \\
13 . \\
26 \\
11 . \\
03 \\
6.9 \\
5\end{array}$ \\
\hline $\begin{array}{l}\mathrm{T}_{2}: \\
\text { Ethrel } \\
300 \\
\text { ppm }\end{array}$ & $\begin{array}{l}13 \\
.9 \\
4\end{array}$ & $\begin{array}{l}19 \\
.5 \\
7\end{array}$ & $\begin{array}{l}21 \\
.6 \\
5\end{array}$ & $\begin{array}{l}21 \\
.2 \\
5\end{array}$ & $\begin{array}{l}73 \\
.1\end{array}$ & $\begin{array}{l}7 \\
3 . \\
4\end{array}$ & $\begin{array}{r}74 \\
.2\end{array}$ & $\begin{array}{l}7 \\
4 . \\
7\end{array}$ & $\begin{array}{l}0 . \\
4 \\
2\end{array}$ & $\begin{array}{l}0 . \\
3 \\
3\end{array}$ & $\begin{array}{l}0 . \\
2 \\
7\end{array}$ & $\begin{array}{l}0 . \\
2 \\
2\end{array}$ & $\begin{array}{l}5 . \\
2 \\
1\end{array}$ & $\begin{array}{c}9 . \\
35\end{array}$ & $\begin{array}{l}10 \\
.7 \\
2\end{array}$ & $\begin{array}{l}17 \\
.7 \\
9\end{array}$ & $\begin{array}{l}4 . \\
9 \\
1\end{array}$ & $\begin{array}{l}6 . \\
8 \\
4\end{array}$ & $\begin{array}{l}8 . \\
3 \\
9\end{array}$ & $\begin{array}{l}11 \\
.3 \\
5\end{array}$ & $\begin{array}{l}16 . \\
26 \\
12 . \\
73 \\
9.4 \\
3 \\
6.0 \\
1\end{array}$ \\
\hline $\begin{array}{l}\mathrm{T}_{3}: \\
\text { Ethrel } \\
400 \\
\text { ppm }\end{array}$ & $\begin{array}{l}14 \\
.1 \\
7\end{array}$ & $\begin{array}{l}20 \\
.7 \\
8\end{array}$ & $\begin{array}{l}21 \\
.9 \\
7\end{array}$ & $\begin{array}{l}21 \\
.5 \\
8\end{array}$ & $\begin{array}{l}73 \\
.7\end{array}$ & $\begin{array}{l}7 \\
3 . \\
9\end{array}$ & $\begin{array}{l}74 \\
.3\end{array}$ & $\begin{array}{l}7 \\
4 . \\
6\end{array}$ & $\begin{array}{l}0 . \\
3 \\
9\end{array}$ & $\begin{array}{l}0 . \\
3 \\
1\end{array}$ & $\begin{array}{l}0 . \\
2 \\
6\end{array}$ & $\begin{array}{l}0 . \\
2 \\
1\end{array}$ & $\begin{array}{l}6 . \\
1 \\
4\end{array}$ & $\begin{array}{l}10 \\
.1\end{array}$ & $\begin{array}{l}10 \\
.9 \\
3\end{array}$ & $\begin{array}{l}17 \\
.8 \\
2\end{array}$ & $\begin{array}{l}5 . \\
2 \\
5\end{array}$ & $\begin{array}{l}7 . \\
3 \\
9\end{array}$ & $\begin{array}{l}8 . \\
4 \\
5\end{array}$ & $\begin{array}{l}11 \\
.4 \\
5\end{array}$ & $\begin{array}{l}15 . \\
03 \\
11 . \\
43 \\
8.2 \\
1 \\
5.0 \\
7\end{array}$ \\
\hline $\begin{array}{l}\text { T4: } \\
\text { Ethrel } \\
600 \\
\text { ppm }\end{array}$ & $\begin{array}{l}14 \\
.9 \\
3\end{array}$ & $\begin{array}{l}21 \\
.1 \\
5\end{array}$ & $\begin{array}{l}22 \\
.8 \\
0\end{array}$ & $\begin{array}{l}21 \\
.6 \\
8\end{array}$ & $\begin{array}{c}74 \\
.2\end{array}$ & $\begin{array}{l}7 \\
4 . \\
7\end{array}$ & $\begin{array}{l}75 \\
.1\end{array}$ & $\begin{array}{l}7 \\
6 . \\
6\end{array}$ & $\begin{array}{l}0 . \\
3 \\
6\end{array}$ & $\begin{array}{l}0 . \\
2 \\
9\end{array}$ & $\begin{array}{l}0 . \\
2 \\
3\end{array}$ & $\begin{array}{l}0 . \\
1 \\
8\end{array}$ & $\begin{array}{l}6 . \\
2 \\
5\end{array}$ & $\begin{array}{l}10 \\
.6 \\
9\end{array}$ & $\begin{array}{l}11 \\
.1 \\
2\end{array}$ & $\begin{array}{l}18 \\
.0 \\
9\end{array}$ & $\begin{array}{l}5 . \\
9 \\
6\end{array}$ & $\begin{array}{l}7 . \\
8 \\
2\end{array}$ & $\begin{array}{l}9 . \\
1 \\
5\end{array}$ & $\begin{array}{l}12 \\
.1 \\
0\end{array}$ & $\begin{array}{l}14 . \\
56 \\
10 . \\
03 \\
6.9 \\
9 \\
3.0 \\
1\end{array}$ \\
\hline $\begin{array}{l}\mathrm{T}_{5}: \\
\text { Cold } \\
\text { water } \\
+ \text { +ethr } \\
\text { el } 200 \\
\text { ppm }\end{array}$ & $\begin{array}{l}12 \\
.9 \\
5\end{array}$ & $\begin{array}{l}19 \\
.5 \\
0\end{array}$ & $\begin{array}{l}21 \\
.9 \\
0\end{array}$ & $\begin{array}{l}21 \\
.1 \\
8\end{array}$ & $\begin{array}{l}72 \\
.3\end{array}$ & $\begin{array}{l}7 \\
2 . \\
8\end{array}$ & $\begin{array}{l}74 \\
.1\end{array}$ & $\begin{array}{l}7 \\
3 . \\
8\end{array}$ & $\begin{array}{l}0 . \\
4 \\
2\end{array}$ & $\begin{array}{l}0 . \\
3 \\
4\end{array}$ & $\begin{array}{l}0 . \\
2 \\
8\end{array}$ & $\begin{array}{l}0 . \\
2 \\
4\end{array}$ & $\begin{array}{l}4 . \\
9 \\
0\end{array}$ & $\begin{array}{l}8 . \\
90\end{array}$ & $\begin{array}{l}10 \\
.6 \\
9\end{array}$ & $\begin{array}{l}17 \\
.5 \\
9\end{array}$ & $\begin{array}{l}4 . \\
8 \\
7\end{array}$ & $\begin{array}{l}6 . \\
8 \\
0\end{array}$ & $\begin{array}{l}8 . \\
3 \\
5\end{array}$ & $\begin{array}{l}11 \\
.3 \\
0\end{array}$ & $\begin{array}{l}16 . \\
94 \\
12 . \\
97 \\
10 . \\
07 \\
6.4 \\
4\end{array}$ \\
\hline $\begin{array}{l}\mathrm{T}_{6}: \\
\text { Hot } \\
\text { water } \\
+ \\
\text { ethrel } \\
200 \\
\text { ppm }\end{array}$ & $\begin{array}{l}12 \\
.4 \\
8\end{array}$ & $\begin{array}{l}19 \\
.2 \\
4\end{array}$ & $\begin{array}{l}21 \\
.0 \\
0\end{array}$ & $\begin{array}{l}20 \\
.7 \\
8\end{array}$ & $\begin{array}{l}72 \\
.1\end{array}$ & $\begin{array}{l}7 \\
2 . \\
5\end{array}$ & $\begin{array}{l}72 \\
.9 \\
9\end{array}$ & $\begin{array}{l}7 \\
3 . \\
3\end{array}$ & $\begin{array}{l}0 . \\
4 \\
6\end{array}$ & $\begin{array}{l}0 . \\
3 \\
5\end{array}$ & $\begin{array}{l}0 . \\
2 \\
9\end{array}$ & $\begin{array}{l}0 . \\
2 \\
5\end{array}$ & $\begin{array}{l}4 . \\
8 \\
7\end{array}$ & $\begin{array}{l}8 . \\
60\end{array}$ & $\begin{array}{l}10 \\
.4 \\
2\end{array}$ & $\begin{array}{l}17 \\
.1 \\
0\end{array}$ & $\begin{array}{l}4 . \\
7 \\
8\end{array}$ & $\begin{array}{l}6 . \\
6 \\
4\end{array}$ & $\begin{array}{l}8 . \\
2 \\
6\end{array}$ & $\begin{array}{l}11 \\
.0 \\
5\end{array}$ & $\begin{array}{l}16 . \\
97 \\
13 . \\
04 \\
10 . \\
32 \\
6.5 \\
8\end{array}$ \\
\hline $\begin{array}{l}\text { CD } \\
\text { @ 5\% } \\
\text { level }\end{array}$ & $\begin{array}{c}0 . \\
08\end{array}$ & $\begin{array}{c}0 . \\
08\end{array}$ & $\begin{array}{l}0 . \\
06\end{array}$ & $\begin{array}{l}0 . \\
06\end{array}$ & $\begin{array}{l}2 . \\
05\end{array}$ & $\begin{array}{l}\mathrm{N} \\
\mathrm{S}\end{array}$ & $\begin{array}{l}1 . \\
26\end{array}$ & $\begin{array}{l}1 . \\
2 \\
3\end{array}$ & $\begin{array}{l}0 . \\
0 \\
5\end{array}$ & $\begin{array}{l}0 . \\
0 \\
5\end{array}$ & $\begin{array}{l}0 . \\
0 \\
4\end{array}$ & $\begin{array}{l}0 . \\
0 \\
4\end{array}$ & $\begin{array}{l}0 . \\
0 \\
7\end{array}$ & $\begin{array}{l}0 . \\
05\end{array}$ & $\begin{array}{c}0 . \\
05\end{array}$ & $\begin{array}{l}0 . \\
07\end{array}$ & $\begin{array}{l}0 . \\
2 \\
1\end{array}$ & $\begin{array}{l}0 . \\
1 \\
0\end{array}$ & $\begin{array}{l}0 . \\
0 \\
9\end{array}$ & $\begin{array}{c}0 . \\
09\end{array}$ & $\begin{array}{l}0.1 \\
8 \\
0.0 \\
8 \\
0.0\end{array}$ \\
\hline
\end{tabular}




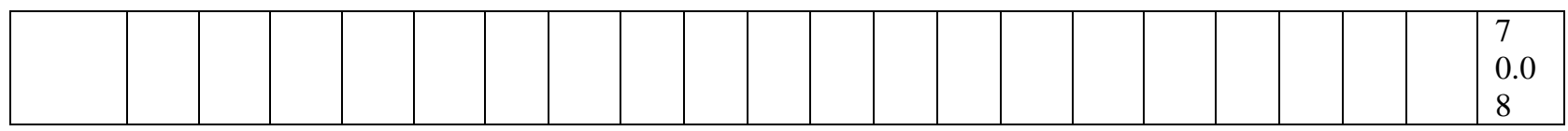

\section{References:-}

1. Akter H, Hassan M K, Rabbani M G and Mahmud A A (2013) Effects of variety and post harvest treatments on shelf life and quality of banana. $J$ Environ sci \& Natural Res 6: 163-175.

2. Deepak G P N, Katiyar J P, Singh and Singh P C (2008) Effect of post harvest chemical treatments on shelf life and physico - chemical quality of banana cv. Harichal. Asian J Hort $3: 386-388$

3. Dharmasena D A N and Kumari A H M R R (2005) Suitability of charcoal- cement passive evaporative cooler for banana ripening. The J of Agri Sci $1: 19-30$.

4. Gowda I N and Hudder A G (2001) Studies on ripening changes in mango fruit. J Food Sci Technol 38 : 135137.

5. Krishnamurthy S (1993) Harvesting handling and storage of fruits. In: Advances in Horticultural Fruit Crops : Part 4 eds Chada, K L and O P Pareek. Malhotra Publishing House, New Delhi, India : 1748-175.7.

6. Kumar P (2006) Effect of packaging methods, temperature and ethylene on shelf life and quality of banana. Msc Thesis, Department of Horticulture and Postharvest Technology Sher - E - Bangla Agricultural University Dhaka : 60.

7. Kulkarni S G, Kudachikar V B and Prakash M N K (2011) Studies on physic - chemical changes during artificial ripening of banana (Musa sp ) variety 'Robusta'. J Food Sci Technol 48 : 730-734.

8. Mahajan B V C, Kaur T, Gill M I S, Dhaliwal B S, Ghuman B S and Chahil B S (2010) Studies on optimization of ripening techniques for banana. J Food Sci Technol 47 : 315-319.

9. Morrelli K L and Kader A A (2002) Recommendations for maintaining postharvest quality. Department of Pomology, University of California, Davis California.

10. Pendharkar P Y, Hiwale S S and Patil H B (2011) Studies on the effect of post harvest treatments on chemical changes during ripening of banana fruits cv. Grand Naine. Internat J Proc \& Post Harvest Technol 2: 32-34.

11. Pillay M and Tripathi L (2007) Banana. Chapter 15. In : C Kole, ed Genome mapping and molecular breeding in plants. Fruits and nuts. Springer - verlag Berlin $4: 281-301$.

12. Pinto A C, Alues R E and Pereira E C (2004) Efficiency of different heat treatment procedures in controlling disease in mango fruits. Proc Seventh Inter Mango Symp Acta Hort 645 : 551-553.

13. Rahman M M and Kabir S M H (2006) Banana. Banglapedia : National Encyclopedia of. Bangladesh.

14. Simmonds N W (1996) Bananas. Lonman Green and Co Ltd London p 466.

15. Singh H P and Chundawat B S (2002) Improved technology of banana. Ministry of Agriculture, Government of India.

16. Temi S, CM ; IDOWU, OA ; IDOWU E (2014) Effect of biological and chemical ripening agents on the nutritional and metal composition of banana (Musa sp.). J Appl Sci Environ Manage 18 : 243-246.

17. Venktasubbaiah K, Jagadeesh S L, Thammaiah N and Chavan M L (2013) Changes in physico - chemical and sensory characteristics of banana fruit cv. Grand Naine during ripening. Karnataka J Agric Sci 26 : 111-114.

18. Zewter A, Woldetsadik K and Workneh T S (2012) Effect of 1- methylcyclopropene, potassium permanganate and packaging on quality of banana. Afr J Agric Res 7 : 2425-2437. 